\title{
IMPORTÂNCIA DO CONHECIMENTO SOBRE DIFERENTES TIPOS DE INTELIGENCIAS NO PROCESSO DE ENSINO-APRENDIZAGEM
}

\author{
Fabiana Galvão da Motta Lima* \\ Luiz Henrique Domingues** \\ Ana Paula Vidotti***
}

RESUMO: O processo de ensino-aprendizagem envolve múltiplos fatores que influenciam na construção do conhecimento pelo indivíduo. A utilização de métodos de ensino tradicionais não enaltece todo o potencial de um aluno, pois valoriza aptidões a apenas algumas competências cognitivas. A teoria das múltiplas inteligências, proposta por Howard Gardner, oferece ferramentas que valorizam diferentes aptidões e o saber multidisciplinar. Diante disso, o objetivo deste trabalho foi avaliar o conhecimento de professores da cidade de Cambará (PR) sobre a teoria e suas aplicabilidades em sala de aula, durante a Operação Rondon Regional 2017 - Norte Pioneiro. A teoria foi apresentada por meio da palestra "Compreendendo o aprendizado: os diferentes tipos de inteligência", inserida na programação da semana pedagógica da cidade, e a avaliação foi feita de forma qualitativa, por observação. Pode-se perceber que a maioria do público-alvo não conhecia a teoria das múltiplas inteligências e apresentavam dificuldades na inserção de atividades pedagógicas não costumeiras ao currículo tradicional. Pode-se então afirmar que há a necessidade de capacitação profissional e reformulação curricular visando à formação na construção de conhecimento pelo indivíduo e não apenas a transmissão de informação.

PALAVRAS-CHAVE: Ensino-Aprendizagem; Operação Rondon Regional 2017; Teoria das Inteligências Múltiplas.

\section{THE IMPORTANCE OF KNOWLEDGE ON DIFFERENT T YPES OF INTELLIGENCE IN THE TEACHING-LEARNING PROCESS}

ABSTRACT: The teaching-learning process involves several factors that affect the agent's construction of knowledge. Traditional teaching methods do not enhance the student's capacity since it gives priority only to certain cognitive competences. The theory of multiple intelligences, proposed by Howard Gardner, provides tools

\footnotetext{
Biomédica. Mestranda no Programa de pós-graduação em Ciências Fisiológicas, Departamento de Ciências Fisiológicas, Universidade Estadual de Maringá (UEM), Brasil. E-mail: affabimottalima@gmail.com

** Discente do curso de Ciências Biológicas, Universidade Estadual de Maringá (UEM), Brasil.

*** Biológa. Docente do Departamento de Ciências Morfológicas (DCM) Universidade Estadual de Maringá (UEM), Brasil.
} 
that valorize different capacities and multidisciplinary knowledge. Current analysis evaluates the knowledge of teachers in Cambará PR Brazil, on the theory and its applicability in the classroom during the Regional Operation Rondon 2017 in the Pioneer Northern region of the state of Paraná, Brazil. The theory was explained by a lecture on "Understand learning: different types of intelligence", within the Pedagogical Week program. Evaluation occurred qualitatively, through observation. Most of the target population was unaware of the theory of multiple intelligences and found it difficult to insert unusual pedagogical activities in the traditional curriculum. Professional capacitation and curricular reformulation are required for the formation of the construction of knowledge by the agent and not merely the transmission of information.

KEY WORDS: Teaching-learning process; Regional Operation Rondon 2017; Theory of multiple intelligences.

\section{INTRODUÇÃO}

O que é a inteligência de um indivíduo? A natureza da inteligência e como defini-la foi avaliada por muitos autores durante anos, com abordagens variando de filosóficas (KEATING, 1984), biológicas, psicométricas a sociais (EYSENCK, 1998). $\mathrm{Na}$ maior parte da história humana, não havia definição científica de inteligência, mas sim o ideal de um indivíduo inteligente: Mahatma Gandhi, Jane Austen, Thomas Jefferson e outras personalidades destacáveis. No entanto, não se fazia a pergunta sobre o que tornava esses indivíduos inteligentes (GARDNER, 2011). Com a ascensão dos métodos psicométricos de mensuração das qualidades cognitivas, destacando-se os testes de QI (coefficient of intelligence) como o método mais conhecido, tornava-se claro um problema: como alguns indivíduos classificados como QI baixo, mediano ou com algum déficit cognitivo de uma forma geral, destacavam-se e obtinham grande sucesso em determinadas áreas do conhecimento (SIEGEL, 1989; LEZAK, 1988).

A resposta para essa pergunta é simples: existem diferentes tipos de competências cognitivas. O neuropsicólogo Howard Gardner, autor do livro Frame of Minds - Theory of Multiple Intelligences (GARDNER, 1983), apresenta a teoria das inteligências múltiplas e propõe a existência de capacidades intelectuais diferentes, 
presentes em todos os indivíduos. Definindo a inteligência como a capacidade de resolução de problemas ou criação de produtos válidos em diferentes aspectos culturais e detalhando critérios a serem considerados como inteligência humana, Gardner propôs inicialmente a existência de sete tipos de competências intelectuais, com maior ou menor aptidão a algumas delas. Atualmente, são propostos nove tipos: naturalista, lógico-matemática, linguística, espacial, corporal-cinestésica, musical, interpessoal, intrapessoal e existencialista (GARDNER, 1989; GARDNER, 1999).

Os métodos psicométricos de avaliação de inteligência falham em avaliar algumas dessas competências, não sendo completamente representativos da capacidade cognitiva do indivíduo. Dessa forma, a teoria das múltiplas inteligências proporciona a criação de métodos de ensino e avaliação não tradicionais, trazendo consigo a possibilidade de melhora do aprendizado individual do aluno (BAS; BEYHAN, 2010; SILVA; NISTA-PICCOLO, 2010; STERNBERG, 2015). Na prática, sabe-se que teorias e diferentes metodologias de ensino funcionam efetivamente se os professores e os alunos colaborarem para fazê-las funcionar. Quando a teoria das múltiplas inteligências é trabalhada e aplicada em sala de aula, o aluno é estimulado de forma positiva, pois suas diferentes aptidões são percebidas e enaltecidas. Transformando o ambiente de ensino em um lugar prazeroso, o professor melhora seu desempenho e torna-se um mecanismo efetivo de transformação social (OLIVEIRA, 2017). Nesse contexto, o presente trabalho demonstra qualitativamente a experiência de conscientização sobre a teoria das múltiplas inteligências de educadores das redes municipal e estadual do município de Cambará (PR).

\section{DESENVOLVIMENTO}

\subsection{PROJETO RONDON E OPERAÇÃO RONDON REGIONAL 2017}

O Projeto Rondon é uma ação voluntária que visa o desenvolvimento sustentável em comunidades necessitadas, desenvolvida pelo Ministério da Defesa, em parceria com governos estaduais, municipais e Instituições de Ensino Superior (IES) públicas e privadas. O projeto beneficia as cidades previamente selecionadas com $\mathrm{o}$ 
envio de professores e universitários de diversas áreas de conhecimento (MINISTÉRIO DA DEFESA, 2017).

A Operação Rondon Regional 2017 foi uma grande ação extensionista realizada na região do Norte Pioneiro do Paraná, durante o período de 23 de julho a 05 de agosto de 2017. Foi coordenada em parceria pelas universidades estaduais de Ponta Grossa (UEPG), do Norte do Paraná (UENP) e do Oeste do Paraná (UNIOESTE), contando com a participação de alunos de 10 instituições de Ensino Superior do Paraná, atuando nas cidades de Jacarezinho, Barra do Jacaré, Cambará, Carlópolis, Conselheiro Mairink, Joaquim Távora, Ribeirão Claro, Santo Antônio da Platina, Siqueira Campos e Wenceslau Brás. A operação foi realizada com o apoio da Secretaria de Ciência, Tecnologia e Ensino Superior (SETI) e da Companhia de Saneamento do Paraná (SANEPAR), conforme os moldes do Projeto Rondon Nacional, com caráter multiplicador e não assistencialista (SETI, 2017; UEPG, 2017).

\subsection{OFICINA "COMPREENDENDO O APRENDIZADO - OS DIFERENTES TIPOS DE INTELIGÊNCIA"}

Voltada para o tema e em forma de palestra seguida de discussão, a oficina foi estruturada para ser parte integrante da Semana Pedagógica do Município, com público-alvo envolvendo professores, pedagogos, diretores de escolas e colégios, entre outros. A organização da programação foi realizada em parceria com a Secretaria de Educação e Cultura da cidade de Cambará e a oficina foi ministrada 3 vezes durante a semana pedagógica.

Inicialmente foram explicados conceitos sobre inteligência - o indivíduo inteligente, demonstrações cotidianas de capacidades cognitivas e usos de testes psicométricos de avaliação intelectual (KEATING, 1984; EYSENCK, 1998; GARDNER, 2011). Foram utilizadas perguntas visando a reflexão pedagógica sobre a distinção que os testes de avaliação infligem sobre os indivíduos, para posterior introdução da teoria das múltiplas inteligências.

$\mathrm{Na}$ sequência, foram explanados os 9 tipos de inteligência propostos por Howard Gardner. Nesse momento, foram introduzidos exemplos de como identificar a aptidão do aluno para o determinado tipo de inteligência e como estimular o desenvolvimento dessa aptidão com atividades escolares que podem ser propostas. 
A inteligência linguística é uma competência ampla, a qual envolve sensibilidade para fala e escrita, além de incluir a habilidade para aprender novos idiomas, construir narrativas e aplicar habilidades metalinguísticas para refletir sobre o uso da linguagem. Já a inteligência lógico-matemática é aquela que denota a capacidade de calcular, quantificar, perceber relações e conexões, utilizar pensamentos abstratos e dedutivos (SANTOS; JUNIOR, 2015; GARDNER, 1989). Ambas são tradicionalmente trabalhadas com maior frequência em sala de aula, mas nem sempre com a inclusão de atividades mais dinâmicas, como por exemplo, a utilização de jogos matemáticos (JUNIOR, 2016; SANTOS; MENDES, 2017; SILVA; SEHN, 2017) e a abordagem da linguística como ferramenta de investigação científica, como jornais (RUIZ; DIAS, 2016; SILVA; RIBEIRO, 2017).

As inteligências interpessoal e intrapessoal dizem respeito a interações humanas. A competência interpessoal é voltada para os outros, sendo que os indivíduos que possuem aptidão para essa inteligência percebem facilmente as interações humanas do ambiente, sendo voltado para liderança, organização e solidariedade. A inteligência intrapessoal diz respeito ao próprio indivíduo, por meio do conhecimento próprio, dos seus sentimentos, objetivos e medos (SANTOS; JUNIOR, 2015; GARDNER, 1999).

Em sala de aula, essas competências podem aparecer de maneiras opostas, e o equilíbrio entre ambas é fundamental e necessário para a personalidade humana, sendo vital o cuidado para não desvalorizar nenhuma das competências. Paralela a essas competências, também há a inteligência existencialista, forte no indivíduo que possui grande sensibilidade para abordar questões profundas sobre a existência humana (GARDNER, 1999). Um exemplo de atividade a ser realizada são os debates com mediadores (SILVA, 2017), estimulando fortemente esses três tipos de inteligência.

A inteligência naturalista condiz com a capacidade de reconhecer características peculiares de seres vivos, bem como a sensibilidade para outras características do mundo natural (SANTOS; JUNIOR, 2015; GARDNER, 1999). É facilmente reconhecida no aluno - curiosidade sobre anatomia e fisiologia humana e/ou animal, plantas, solo, meio ambiente, entre outros. Para estimular esse tipo de competência, considerando sua abrangência, é interessante incluir passeios ao ar livre, visitas a la- 
boratórios de ciências, museus e aulas práticas no cronograma escolar (POZENATO; PACHECO; SCOPEL, 2016; SOUZA, 2017).

A competência musical envolve a capacidade de discernir entre ritmo, timbre e tom, permitindo que o indivíduo atue na composição, performance e apreciação de padrões musicais (SANTOS; JUNIOR, 2015; GARDNER, 1999). Ações e perspectivas para o ensino musical escolar são amplamente discutidas, pois se reconhece a sua necessidade e importância de propostas consistentes de educação musical. No entanto, tais perspectivas não são fortemente aplicadas. Estratégias como percepção de sons cotidianos diversificados, definição da identidade sonora de um contexto cultural, uso de repertórios valorizados pelo estudante, entre outros, são algumas ideias (QUEIROZ; MARINHO, 2009).

A inteligência espacial engloba a capacidade de pensar em três dimensões, de orientar-se em um ambiente concreto e abstrato e relacionar medidas e formas. O indivíduo com aptidão nessa competência possui boa noção de orientação e preferência pela linguagem visual (SANTOS; JUNIOR, 2015; GARDNER, 1999). O uso de tecnologias de informação, comunicação, design e desenho são exemplos de práticas pedagógicas que fortalecem essa inteligência (PLETSCH; SOUZA; ORLEANS, 2017; CARVALHO, 2011).

Por último, a inteligência corporal-cinestésica, a qual designa capacidades de resolver problemas, criar produtos e participar de atividades utilizando as funções motoras. O sujeito que apresenta essa inteligência desenvolvida apresenta grande destreza física e prefere aprender executando a tarefa. A inserção de esportes, aulas de dança, aulas práticas de ciência e outras disciplinas são ferramentas didáticas válidas para o correto desenvolvimento dessa competência intelectual (DAOLIO, 1996; SOUZA; BRANDÃO, 2017).

O fechamento da oficina se deu por debate com a realização de perguntas pelo público-alvo e conversas informais com professores. Opiniões foram colhidas, em forma de pequeno comentário escrito, para feedback da oficina a ser repassado para a coordenação da Operação Rondon Regional 2017. 


\section{RESULTADOS E DISCUSSÃO}

A Operação Rondon Regional 2017 possuiu como principal objetivo a formação de indivíduos multiplicadores entre a população do município atendido. Dessa forma, as ações realizadas passam a ser de efeito duradouro, com benefícios a longo prazo para a população, a economia, o meio ambiente e a administração da cidade. Os acadêmicos participantes se tornam ferramentas de transformação da sociedade. Por meio das oficinas realizadas, pode-se conhecer a verdadeira realidade brasileira e desenvolver um sentimento de responsabilidade social e coletiva, em defesa humana, contribuindo também para a formação acadêmica e pessoal do indivíduo rondonista.

O tripé ensino-pesquisa-extensão fundamenta a universidade brasileira e não deve ser compartimentalizado (MOITA et al., 2009). No entanto, o que se percebe no ambiente universitário é que o aluno se encontra imerso em atividades acadêmicas, muitas vezes voltadas com exclusividade à pesquisa, não as equiparando ao ensino e extensão universitária. Apesar de possuir caráter extensionista, a Operação Rondon Regional 2017 trouxe ferramentas para que o ensino fosse valorizado e para que a pesquisa acadêmica fosse aplicada, aproximando o aluno dos valores primordiais da universidade brasileira e fortalecendo o tripé por meio da participação efetiva do discente em todas as funções.

A oficina atingiu um público-alvo de aproximadamente 200 pessoas. Por observação informal, foi possível observar maior número de participantes do sexo feminino. Houve grande envolvimento dos participantes, por meio de histórias presenciadas em sala de aula ou fazendo perguntas sobre como lidar com situações-problema encontradas durante estruturação de atividade pedagógicas, dentre outras. Constatou-se que os educadores presentes, em sua maioria, não eram familiarizados com a teoria das inteligências múltiplas de Howard Gardner, mas já haviam percebido diferentes aptidões em seus alunos durante atividades escolares. Muitos relataram que a oficina os ajudaria a tornar suas práticas de ensino mais eficientes, como apresentado no seguinte relato anônimo fornecido por um educador: "Uma excelente palestra, que deixou claro os nove tipos de inteligências e a forma que podem ser trabalhadas em sala de aula para o bom desenvolvimento do aluno". 
Há alguns anos, os conhecimentos adquiridos na escola eram carregados pela vida inteira como ideais a serem seguidos e ensinados. Possuir um diploma era sinal de competência vitalícia. No entanto, não há dúvidas que os tempos são outros e a educação agora volta-se para oportunidades para explorar novos aprendizados e atualizações (LIBÂNEO; OLIVEIRA; TOSCHI, 2017). No entanto os problemas existentes na área educacional permanecem os mesmos há tempos, e as ações públicas implementadas falharam em provocar mudanças importantes no processo de ensino-aprendizagem, pois não levaram em consideração como o aluno construía o conhecimento. As aspirações, interesses e expectativas dos alunos estavam sendo deixadas de lado (MORAES, 1996).

Durante os debates pós-oficina, uma situação se repetia: casos frequentes em que alunos eram vistos como "preguiçosos" por não se esforçar tanto em uma disciplina quanto em outra foram reavaliados pelos educadores. Os professores perceberam que, conforme o desenvolver da disciplina, sinais eram dados pelos alunos de que, talvez, determinada prática pedagógica não fosse a melhor na ocasião. No entanto, a comunicação aluno-professor foi falha na grande maioria dos casos. Não havia o conhecimento necessário, tanto por parte docente quanto discente, para argumentar uma solução.

Na prática do ensinar, encontram-se muitos modelos de educação e de instituições fundamentados em determinadas teorias do conhecimento. É importante ressaltar que, simultaneamente, a educação é influenciada pela ciência, e a mesma é influenciada pela educação. O indivíduo humano ensina e difunde o conhecimento por meio da compreensão dos processos inerentes à vida (MORAES, 1996). Nesse momento que é necessária a atualização dos professores: ciência defasada produz uma educação falha, gerando indivíduos incapazes de moldar conhecimento.

Neste contexto, destaca-se a grande necessidade da reformulação do processo de ensino-aprendizado, evoluindo da educação tradicional voltada para a transmissão de conteúdos para uma educação centralizada no indivíduo aluno e em seu processo de aprendizagem. Para tal, são necessárias ferramentas para os educadores conhecerem a relevância de se criar ambientes de aprendizado interdisciplinares, intuitivos e criativos. Além disso, o conhecimento sobre a teoria das múltiplas inteligências também pode ser benéfico ao aluno, que, dessa forma, estaria apto a 
protagonizar seu próprio processo de aprendizado. A oficina realizada durante a Operação Rondon Regional 2017 foi significativa nesse sentido, fornecendo atualizações de práticas de ensino que podem ser implementadas visando a melhoria do trabalho do professor e da educação do indivíduo, como constatado nas conversas e relatos.

Durante o período de realização da Operação Rondon Regional 2017, os feedbacks recebidos sobre a oficina "Compreendendo o aprendizado: os diferentes tipos de inteligência" foram positivos, com conversas sobre a temática acontecendo nas escolas da cidade de Cambará. No entanto, é importante ressaltar que a inserção é um processo frágil, o qual envolve muitas variáveis tanto na escola quanto no corpo docente.

Incorporando-se atividades voltadas à multidisciplinaridade e, consequentemente, às múltiplas competências cognitivas, deve-se manter uma avaliação constante e frequente para que as devidas adaptações sejam realizadas. Dessa forma, a instituição educativa consegue explorar, de maneira efetiva, todo o potencial da aplicação da teoria das inteligências múltiplas. É de fundamental importância que os docentes estejam capacitados para aplicar tais atividades e conceitos; como observado na cidade de Cambará, a maioria dos educadores não conheciam as múltiplas inteligências e demonstraram apresentar dificuldades para utilizar atividades diferenciadas do currículo tradicional. É papel da escola acompanhar e estimular o trabalho docente (BATISTA; ANDRADE; RAMALHO, 2017).

\section{CONSIDERAÇÕES FINAIS}

A participação na Operação Rondon Regional 2017 permitiu grande vivência à equipe participante. Os alunos puderam perceber que a realidade não corresponde completamente ao ensinado em sala de aula e tiveram a oportunidade de estruturar estratégias para lidar com essas diferenças, característica que torna o aluno mais humano, empático e social.

A criação da oficina "Compreendendo o aprendizado: os diferentes tipos de inteligência" originou-se do desejo de capacitar educadores para tornar as ativi- 
dades de sala de aula mais atrativas e efetivas aos alunos, propondo que os mesmos utilizassem a teoria das múltiplas inteligências como base. Um grande problema enraizado na educação brasileira é a falta de interesse do aluno, e muitos debates ocorrem sobre sua origem. Os feedbacks recebidos após a realização das oficinas tornaram claro para a equipe que o potencial pedagógico da teoria para a resolução desse problema é enorme, mas que sim, existem falhas. Sem a devida capacitação, e busca efetiva por ferramentas adequadas de auxílio pedagógico, sua aplicação é dificultada.

Diante disso, pode-se perceber que o primeiro passo já foi dado para que a inserção de atividades pedagógicas visando a teoria das inteligências múltiplas aconteça. Acredita-se que o próximo passo deva ser dado pelos educadores, buscando meios de utilizar a teoria na prática em suas escolas, investigando onde implantar, como implantar e, mais importante, como solucionar possíveis empecilhos durante esse processo. Sua total aplicação é trabalhosa, sendo importante a estruturação de uma estratégia prévia para resolução de problemas.

\section{REFERÊNCIAS}

BAS, G.; BEYHAN, Ö. Effects of multiple-intelligence supported project-based learning on students' achievement levels and attitude towards English lesson. International Electronic Journal of Elementary Education. v. 2, n. 10, p. 365-386, 2010.

BATISTA, C.G.; ANDRADE, P.C.R.; RAMALHO, M.L. As tecnologias na educação e a interface com o currículo de um curso de uma instituição tecnológica. Revista CESUMAR. v. 22, n. 2, p. 63-75, 2017.

DAOLIO, J. Educação Física escolar: em busca da pluralidade. Revista Paulista de Educação Física. supl. 2, p. 40-42, 1996.

EYSENCK, H.J. The Concept of "Intelligence": Useful or Useless? Intelligence. v. 12, p. $1-16,1988$.

GARDNER, H. Frame of Minds: The Theory of Multiple Intelligences. New York: Basic Book, 2011. 
GARDNER, H; HATCH, T. Multiple Intelligences Go to School: Educational Implications of the Theory of Multiple Intelligences. Educational Researcher. v. 18, n. 8, p. 4-10, 1989.

GARDNER, H. The Intelligence reframed: multiple intelligences for the 21st century. New York: Basic Book, 1999.

MÉDICO JUNIOR, J.L. Jogos de Aplicação Didática nas Aulas de Matemática. 2016. 29f. Trabalho de Conclusão de Curso (Licenciatura Plena em Matemática) Universidade Federal de São João Del Rei, Serrana, 2016.

LEZAK, M. IQ: R.I.P. Journal of Clinical and Experimental Neuropsychology. v. 10, n. 3, p. 351-361, 1988.

LIBÂNEO, J.C.; OLIVEIRA, J.F.; TOSCHI, M. S. Educação escolar: políticas, estruturas e organização. São Paulo: Cortez, 2017.

MENDES, I.A. História para o ensino da matemática: uma reinvenção didática para a sala de aula. Revista Cocar, n. 3, Edição especial, p. 145-166, 2017.

MINISTÉRIO DA DEFESA. Projeto Rondon. Disponível em: < http://www.defesa. gov.br/programas-sociais/projeto-rondon > . Acesso em: 10 jan. 2018.

MORAES, M.C. O paradigma educacional emergente: implicações na formação do professor e nas práticas pedagógicas. Em aberto. v. 16, n. 70, p. 58-69, 1996.

MOITA, G.S.C. et al. Ensino-pesquisa-extensão: um exercício de indissociabilidade na pós-graduação. Revista Brasileira de Educação. v. 14, n. 41, p. 269-280, 2009.

OLIVEIRA, B.N. Aplicações em sala de aula da Teoria das Inteligências Múltiplas. 2017. 57f. Tese (Mestrado em Matemática) - Pontifícia Universidade Católica do Rio de Janeiro, Rio de Janeiro, 2017.

PLETSCH, M.D.; SOUZA, F.F.; ORLEANS, L.F. A diferenciação curricular e o desenho universal na aprendizagem como princípios para a inclusão escolar. Revista Educação e Cultura Contemporânea. v. 14, n. 35, 264-281, 2017. 
POZENATO, A.; PACHECO, G.; SCOPEL, J.M. et al. A importância da visitação monitorada no Museu de Ciências Naturais da Universidade de Caxias do Sul. Scientia Cum Industria. v. 4, n. 4, p. 244-247, 2016.

QUEIROZ, L.R.S.; MARINHO, V.M. Práticas para o ensino da música nas escolas de educação básica. Música na educação básica. Porto Alegre, v. 1, n. 1, p. 60-75, 2009.

RUIZ, E.M.S.D.; DIAS, A.V.M. Gramática e Gênero de Discurso: novas perspectivas para o ensino de língua portuguesa. Signum: Estudos da Linguagem. v. 19, n. 1, p. 241-266, 2016.

SANTOS, N.F.; MENDES, A.A. Alunos com baixa visão: atividades pedagógicas e estratégias de aprendizagem na matemática. In: II SEMINÁRIO CIENTÍFICO DA FACIG E I JORNADA DE INICIAÇÃO CIENTÍ́FICA DA FACIG, 2016. Anais... 2016.

SANTOS, T.F.S.; BOTTENTUIT JUNIOR, J.B. Perspectivas da Teoria das Inteligências Múltiplas para a Educação a Distância: um estudo no curso de licenciatura em pedagogia do NEAD-UFMA. Revista EducaOnline. v. 9, n. 1, p. 68-102, 2015.

SECRETARIA DA CIÊNCIA, TECNOLOGIA E ENSINO SUPERIOR. Operação Rondon 2017 começa dia 23 de julho, 2017. Disponível em: < http://www.seti.pr.gov.br/ modules/noticias/article.php?storyid=5407> . Acesso em: 11 jan. 2018.

SIEGEL, L. IQ is irrelevant to the definition of learning disabilities. Journal of Learning Disabilities. v. 22, n. 8, p. 469-476, 1989.

SILVA, C.P. O ensino de história e o uso de metodologias diferenciadas em sala de aula: o relato de uma experiência no ensino fundamental e médio. 2017. 16f. Trabalho de Conclusão de Curso (Licenciatura em História) - Universidade Estadual da Paraíba, Guarabira, 2017.

SILVA, J.A.M.; RIBEIRO, J.S.M. O Jornal como Ferramenta Didática. Revista Eletrônica Científica Inovação e Tecnologia. v. 3, n. 17, 2017. 
SILVA, R.X.; SEHN, E. A utilização dos jogos na alfabetização matemática. Revista Eletrônica Científica Inovação e Tecnologia. v. 3, n. 17, 2017.

SILVA, V.L.T.; NISTA-PICCOLO, V.L. Dificuldade de aprendizagem na perspectiva das inteligências múltiplas: um estudo com um grupo de crianças brasileiras. Revista Portuguesa de Educação. v. 23, n. 2, p. 191-211, 2010.

SOUZA, L.W.; BRANDÃO, H.C. Aulas práticas e sua importância no ensino da Biologia. Revista Eletrônica Científica Inovação e Tecnologia. v. 3, n. 17, 2017.

STERNBERG, R.J. Multiple Intelligences in the New Age of Thinking. In: GOLDSTEIN, S.; PRINCIOTTA, D.; NAGLIERI, J. (Ed.) Handbook of Intelligence. New York: Springer, 2015, p. 229-241.

UNIVERSIDADE ESTADUAL DE PONTA GROSSA. Operação Rondon 2017 se despede do Norte Pioneiro, 2017. Disponível em: < http://portal.uepg.br/noticias. php?id=11078> . Acesso em: 15 jan. 2018.

Recebido em: 14/03/2018 Aceito em: 16/04/2018 